\title{
Archéopages
}

Archéopages

Archéologie et société

$43 \mid 2016$

Médecines

\section{Se soigner à Fanum Martis dans l'Antiquité}

Les enseignements de l'instrumentum

Healing at Fanum Martis in Antiquity. The teachings of Instrumentum

Sanarse en Fanum Martis en la Antigüedad. Las enseñanzas del

instrumentum

Bérangère Fort, Nicolas Tisserand, Jennifer Clerget et Raphaël Clotuche

\section{OpenEdition}

\section{Journals}

Édition électronique

URL : https://journals.openedition.org/archeopages/1901

DOI : 10.4000/archeopages. 1901

ISSN : 2269-9872

Éditeur

INRAP - Institut national de recherches archéologiques préventives

Édition imprimée

Date de publication : 1 novembre 2016

Pagination : $34-43$

ISSN : $1622-8545$

\section{Référence électronique}

Bérangère Fort, Nicolas Tisserand, Jennifer Clerget et Raphaël Clotuche, « Se soigner à Fanum Martis dans l'Antiquité », Archéopages [En ligne], 43 | 2016, mis en ligne le 01 décembre 2018, consulté le 02 juin 2021. URL : http://journals.openedition.org/archeopages/1901 ; DOI : https://doi.org/10.4000/ archeopages. 1901 


\section{Se soigner à Fanum Martis dans l'Antiquité \\ Les enseignements de l'instrumentum}

Bérangère Fort Inrap, UMR 6298 "ArTeHiS "

Nicolas Tisserand Inrap, UмR 6298 "ArTeHiS"

avec la collaboration de Jennifer Clerget Inrap

Raphaël Clotuche Inrap, UMR 7041 «ArSCAn"

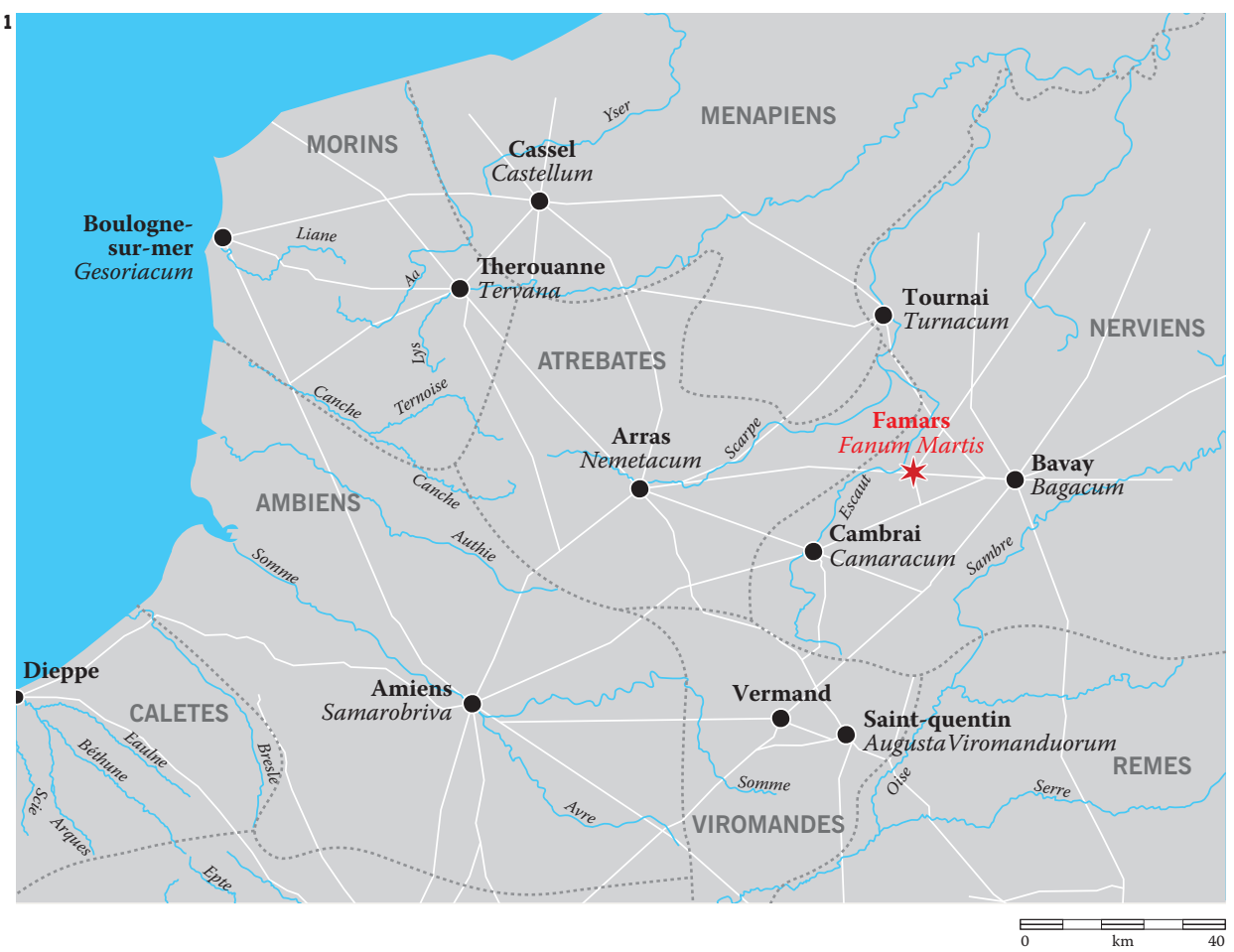

- Nécropoles

- Voies antiques

Limites de l'espace urbain

au Haut - Empire

Parcelles diagnostiquées

ou fouillées

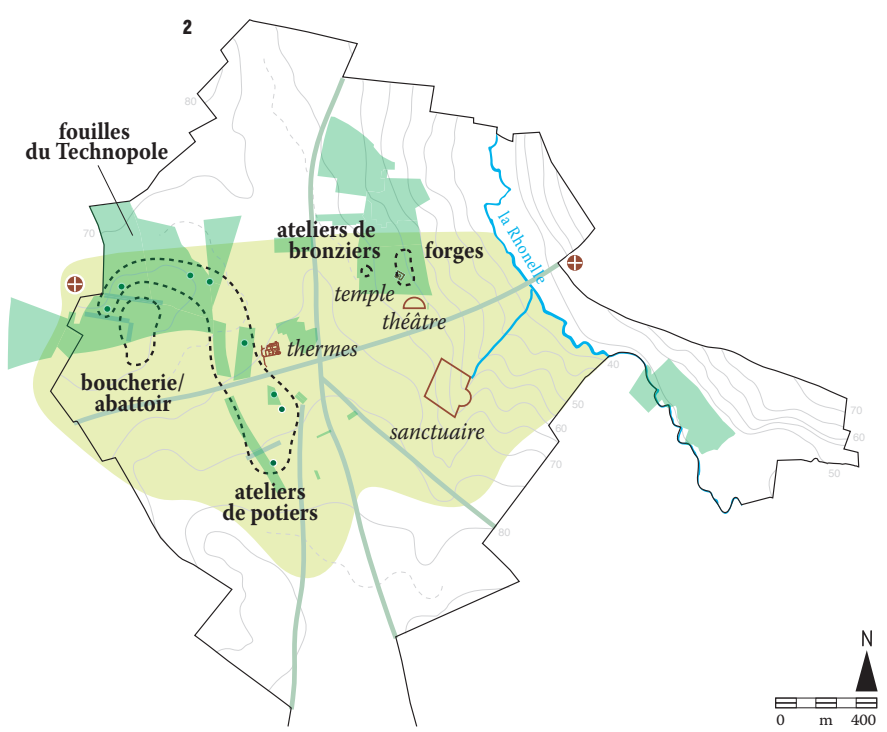

1. Fanum Martis était positionnée en plein cœur d'un dense réseau

d'agglomérations, sur

une butte dominant la rivière

Escaut qui sépare les

territoires nervien et

atrébate.

2. L'agglomération de Fanum

Martis couvrait à son apogée,

à la fin du III siècle, une

superficie de près de 150 ha

Les fouilles du Technopole,

dans la partie ouest

de l'agglomération, ont

mis en évidence un quartier

d'habitat à vocation

artisanale. 
Pour les Romains, à la suite des Grecs et Hippocrate, conserver la santé, c'est réguler les humeurs (chaude, froide, sèche, humide) qui régissent le fonctionnement du corps ; cela passe par une alimentation équilibrée, par la prise de remèdes naturels, souvent phytothérapiques, par la chirurgie, par l'hygiène et par les croyances.

Il est parfois difficile de mettre en lumière certaines de ces pratiques qui laissent peu de traces. Les lieux de soin sont multiples : sphère domestique ou privée pour les soins hygiéniques et cosmétiques, voire pour certains gestes médicaux (visites de médecin, visites chez le médecin...) et sphère publique avec les thermes et les sanctuaires où sont dispensés des soins. Les hôpitaux sont réservés quant à eux à la sphère militaire avec les valetudinaria des camps. On sait par ailleurs que les médecins étaient souvent ambulants, réalisant des visites de ville en ville, tel l'oculiste d'Augst/Augusta Raurica (Suisse) par exemple (Hirt, 2000, p. 109). Les nécropoles permettent quant à elles, en livrant des données paléopathologiques, de déterminer l'état sanitaire des populations. Elles peuvent en outre livrer des tombes dont le mobilier indique des activités médicales ou des activités tournées vers la toilette et la cosmétique.

Les sources archéologiques de ces pratiques peuvent donc être ténues et les indices matériels dont nous disposons sont parfois difficiles à interpréter (Gourevitch, 2011). Par conséquent, les « petits » objets constituent de bons indicateurs des soins pratiqués au quotidien et constituent bien souvent les seuls indices dont nous disposons pour éclairer ces questions de soin et d'hygiène qui constituent les bases de la santé. Ainsi, le corpus des objets de soin découverts sur l'agglomération secondaire de Famars/Fanum Martis, relativement conséquent (163 objets), offre des perspectives intéressantes quant au soin et à la façon de soigner en Gaule Belgique au cours des quatre premiers siècles de notre ère.

\section{Les fouilles de Fanum Martis et le corpus mis au jour}

Connue de longue date, l'agglomération de Famars/Fanum Martis fait l'objet d'une attention particulière depuis une dizaine d'années grâce à la réalisation de fouilles préventives de grande ampleur, notamment celles du Technopole ${ }^{\mathbf{1}}$, avec, en parallèle, la création d'un Projet collectif de recherche $(\mathrm{PCR})$ permettant de faire la synthèse

1. Dirigées entre 2011 et 2014 par Jennifer Clerget et Géraldine Teysseire, Inrap.

2. L'ensemble de ces

travaux est mené sous

la direction de Raphaël

Clotuche, Inrap.

3. Dans ce lot, 19187

individus correspondent

à la clouterie, à la

quincaillerie et aux

indéterminés.

4. Ce travail est envisagé au sein du PCR. nombreuses données désormais disponibles. en marge des grands axes de communication terrestres, mais est positionnée entre deux cours d'eau navigables, l'Escaut et la Rhonelle, qui lui permettent des relations commerciales nourries [ill. 1].

L'origine de l'agglomération demeure mal connue, bien que des indices ponctuels d'une lagglomération antique de Famars se trouve occupation laténienne soient perceptibles. Tirant son nom d'un temple (non fouillé) dédié à Mars, Famars connaît un développement rapide dans le courant de la seconde moitié du I $^{\mathrm{er}}$ siècle de notre ère. Dans le courant de la première moitié $\mathrm{du} \mathrm{IV}^{\mathrm{e}}$ siècle, un castrum vient fortifier le centre de l'agglomération. Celle-ci marque alors un net repli. Dans le courant du haut Moyen Âge, elle décline au profit de la ville de Valenciennes (Clotuche, 2016) [ill. 2].

La reprise des collections d'objets découverts plus ou moins anciennement à Famars et l'étude de ceux mis au jour au cours des fouilles du Technopole constituent un corpus important quasiment inégalé pour une agglomération de taille similaire, qui n'est pas, rappelons-le, une capitale de cité. Au sein des 21 054 objets étudiés (hors céramique), 163 peuvent être rattachés aux soins du corps ${ }^{3}$. Ceux issus des fouilles récentes du Technopole (109 NMI) sont les mieux documentés, notamment du point de vue chronologique. Ils ont également pu faire l'objet d'une analyse spatiale par le biais d'un système d'information géographique (SIG), permettant d'appréhender les questions de caractérisation des espaces au sein d'un quartier et celles des assemblages d'objets. Les objets découverts plus anciennement (54 NMI) n'ont pas encore pu être intégrés au $\mathrm{SIG}^{\mathbf{4}}$ ou ne pourront jamais y être intégrés faute de localisation précise. Nos problématiques spatiales sont donc centrées, dans un premier temps du moins, sur les zones les mieux renseignées.

\section{Les matériaux du corpus}

L'examen de la répartition des matériaux mis en ouvre pour la fabrication des 163 objets de soin de Famars [ill. 3] offre une image paradoxalement attendue et inhabituelle. On note une surreprésentation des alliages cuivreux qui constituent les trois quarts du corpus ainsi qu'une bonne représentation des objets en verre (20 \%). Les autres matériaux ne sont attestés que de façon anecdotique : le fer ne représente que $2 \%$ de l'ensemble, les objets en pierre $1 \%$, alors que les matières dures animales sont totalement absentes. La forte proportion d'objets en alliage cuivreux, attestée sur d'autres sites comme ceux de Lyon par exemple (Vigier, 2015, fig. 3), n'est pas surprenante. Elle peut être expliquée par le fait que les alliages cuivreux sont les mieux adaptés pour les soins, à la fois solides, permettant la mise en œuvre de formes fines et surtout peu soumis à la corrosion, contrairement au fer, ce qui les rend beaucoup plus « hygiéniques ». Celse (Aulus Cornelius Celcus) par exemple, au $\mathrm{II}^{\mathrm{e}}$ siècle de notre ère dans son Traité de la Médecine, cite l'airain, un alliage cuivreux, comme le matériau dédié aux sondes auriculaires dont l'usage est très polyvalent: «On se procure donc des sondes d'airain [...] » (livre VII, chap. XXVI, \$1). L'utilisation des alliages cuivreux comme matériau de prédilection pour

\section{$\stackrel{m}{\mu}$



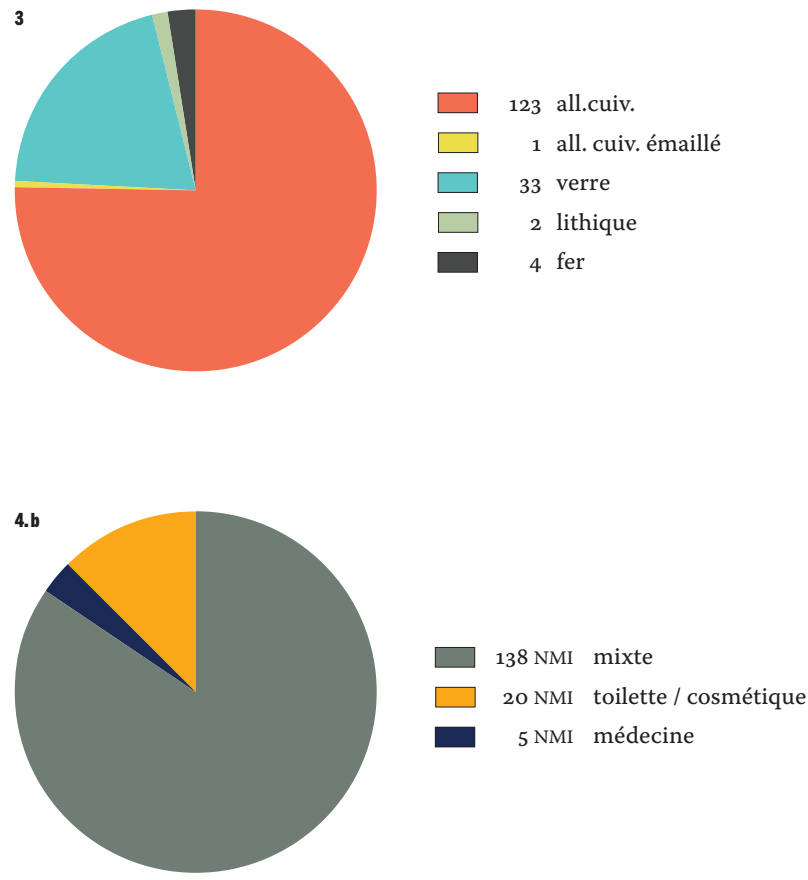

\begin{tabular}{|l|l|l|}
\hline 4.a & NMI \\
\hline Toilette / cosmétique & forces & 2 \\
\hline miroir & 14 \\
\hline nécessaire de toilette & 2 \\
\hline strigile & 1 \\
\hline vase à parfum & 1 \\
\hline Mixte & agitateur & 7 \\
\hline cuillère-sonde & pincette & 21 \\
\hline récipient en verre & 8 \\
\hline sotal & sonde à spatule & 29 \\
\hline sonde auriculaire & 3 \\
\hline sonde fragmentaire & 56 \\
\hline tablette à broyer & 5 \\
\hline tige d'instrument & 7 \\
\hline érigne ? & 1 \\
\hline instrument à palette & 3 \\
\hline sonde médicale & 1 \\
\hline & & 2 \\
\hline
\end{tabular}

les objets de soin non tranchants est donc connue et reconnue avant l'invention de l'acier inoxydable dont ils sont composés aujourd'hui.

Quant à la faible proportion d'objets en fer, elle trouve une explication en négatif de la forte représentation des objets en alliages cuivreux. Il convient néanmoins de nuancer cette image : il est fort probable que nombre d'objets en fer présentant des tranchants tels que rasoirs ou scalpels ne soient pas parvenus jusqu'à nous ou qu'ils n'aient pu être identifiés. En effet, ces objets de dimensions relativement faibles présentant des tranchants très fins subissent de fortes altérations et ne sont soit plus conservés, soit plus identifiables. Parallèlement, il est fort probable qu'un certain nombre de petites lames en fer très abîmées et classées au sein des objets d'usage polyvalent correspondent à des objets de soin. Il convient donc de garder à l'esprit ce biais accentué pour les découvertes anciennes où seuls les « beaux objets » étaient conservés. La très faible portion des objets en fer de Lyon (moins de $1 \%$ ), encore plus faible que celle de Famars, peut très certainement être expliquée par des phénomènes analogues.

À Famars, le verre est très bien représenté, à la cosmétique et aux remèdes ainsi que dans celle de certains objets tels que les agitateurs. Si nous avons choisi ici de conserver les petits récipients en verre ${ }^{\mathbf{5}}$, cette option n'a pas été retenue à Lyon où Élise Vigier considère que ces objets sont d'usage trop polyvalent pour appartenir aux objets de soin (Vigier, 2015, p. 578). Nous considérons cependant que les petits flacons de type balsamaire ou aryballe et les petits pots correspondaient prioritairement à un usage cosmétique, hygiénique voire médical (Bertrand, 2003). Notre corpus est donc agrémenté des petits récipients en verre, souvent très fragmentaires et majoritairement issus des fouilles récentes, les fragments des fouilles anciennes n'étant pas conservés.

Dans notre corpus, l'usage de la pierre est réservé à la fabrication des rares tablettes à broyer identifiées. Aucun petit mortier ou pilon en pierre n'a été reconnu. Néanmoins, les objets en pierre, souvent fragmentaires, sont parfois difficiles à reconnaître. Sans analyse fine, on peut facilement confondre une tablette à broyer avec un élément de placage ou un pilon avec un outil à abraser.

Par ailleurs, l'absence totale d'objets réalisés en matières dures d'origine animale est très surprenante. Alors qu'à Lyon ceux-ci constituent près de $24 \%$ du corpus, aucun objet n'a été identifié 
à Famars malgré une étude spécifique de ces matériaux ${ }^{6}$. Seuls deux peignes en os découverts anciennement sont mentionnés dans la Carte archéologique de la Gaule (Delmaire, 1996), mais n'ont pas été retenus puisque issus de découvertes réalisées dans la zone des grands thermes publics et du castrum, secteur qui a aussi livré des sépultures du haut Moyen Âge ; leur attribution chronologique trop incertaine et leur absence des collections du Musée de Valenciennes nous ont conduits à les écarter du corpus. Cette absence d'objets en matières dures animales est tout à fait surprenante, d'autant que les fouilles du Technopole ont porté sur un quartier artisanal travaillant entre autres les matières animales, de la boucherie à la colle d'os en passant par la tabletterie, la matière première ne manquait donc pas. Cette absence ne trouve à ce jour aucune explication autre que celle du hasard ou d'un goût peu marqué à Famars pour l'usage des matières dures animales dans le domaine des soins.

Enfin, un certain nombre de gestes d'hygiène du quotidien nous échappent complètement car ils étaient réalisés avec des objets qui ne laissent pas de traces ; le brossage des dents, par exemple, pouvait tout à fait être réalisé avec des fibres végétales. En outre, aucun objet en bois n'a été découvert, bien que nous sachions que ce matériau était fréquemment employé dans la fabrication de peignes et de petits récipients tels des pyxides qui pouvaient contenir des onguents et qu'il devait être aussi utilisé pour les cure-dents et autres petits instruments pointus.

\section{La cosmétique et les soins médicaux}

Trois catégories fonctionnelles ont pu être distinguées : la catégorie des objets de toilette et de cosmétique, celle de l'instrumentum purement médical et celle dont l'usage est mixte et qui regroupe des objets polyvalents, utilisés à la fois dans les soins quotidiens relevant de l'hygiène et de la cosmétique, mais qui peuvent également être employés pour des soins médicaux [ill. 4a et b].

\section{La toilette}

La catégorie de la toilette et de la cosmétique est illustrée par 20 objets dont l'usage ne peut être autre [ill. 5]. On trouve ainsi des forces dont les faibles dimensions les recommandent pour la toilette (entretien de la barbe ou des cheveux). Deux objets (pincette et cure-oreilles) dotés d'un anneau de suspension appartiennent à des nécessaires de toilette, petits trousseaux personnels que l'on emporte avec soi, bien connus durant l'Antiquité. Des nécessaires tout à fait similaires sont d'ailleurs encore en usage à l'image de nos coupe-ongles dotés d'une lime ou des nécessaires de poche contenant ciseaux, pinces, limes... Morphologiquement plus proche des modèles romains, nous pouvons citer un parallèle ethnographique contemporain provenant du Népal [ill. 6]. La pincette de Famars (M-1241) est cependant curieuse car réalisée en fer, matériau qui, on l'a vu, n'est pas très adapté aux objets de soin. Un strigile (racloir utilisé pour la toilette), en fer lui aussi, très fragmentaire a été identifié. Les strigiles en fer sont plutôt rares, mais des découvertes récentes issues de l'archéologie préventive viennent compléter nos données (Mallet, 2009, p. 113-152 ; Chevrier, en cours). Ainsi, un examen ou un réexamen de certains fragments d'objets en fer pourraient compléter ce corpus d'objets encore mal identifiés. Les objets de toilette les mieux représentés à Famars sont les miroirs. Même fragmentaires, ces objets plats en alliage cuivreux à forte teneur en étain, soigneusement polis sur au moins l'une des faces, voire sur les deux, sont très caractéristiques. Pline l'Ancien, dans son Histoire Naturelle, au livre XXXIII traitant des métaux, l'explique lui aussi : "Pour en finir ici avec les miroirs, ajoutons que les meilleurs chez nos ancêtres étaient ceux de Brindes, formés d'un mélange d'étain et de cuivre [...] » (chap. XLV, $\mathbb{S I X ) . ~ O u t r e ~ l e s ~ f r a g m e n t s ~ d e s ~ m i r o i r s ~}$ eux-mêmes, qu'ils soient circulaires ou rectangulaires, décorés ou non, on trouve un manche peu soigné, composé d'une tige de section circulaire en forme de balustre. Ces 14 éléments de miroirs de Famars attestent de l'usage fréquent de ces objets, vraisemblablement par toutes les catégories sociales. Enfin, un vase à parfum en alliage cuivreux émaillé, découvert anciennement et conservé au Musée du Louvre, constitue quant à lui un bel exemple d'objet luxueux. Le taux de représentation d'objets de toilette, $12 \%$ du corpus à Famars, est tout à fait comparable à celui observé à Augst/Augusta Raurica par exemple où ils forment 13,4\% du corpus des objets de soins, de toilette et de médecine (Riha, 1986).

\section{Les objets à usage mixte}

Les objets d'usage mixte sont de loin les plus nombreux (138 NMI), comme à Lyon ou à Augst (Vigier, 2015 ; Riha, 1986). Ils sont aussi très variés [ill. 7]. On trouve une trentaine de récipients en verre : aryballes, balsamaires, petits flacons et petits pots servant à contenir des parfums ou des onguents. Ces éléments sont très fragmentaires, mais leur identification est possible. On reconnaît également des fragments d'agitateurs en verre et en alliage cuivreux qui servent à mélanger des préparations cosmétiques ou médicales. Pour les exemplaires en métal, seules les statuettes zoomorphes qui les surmontaient sont conservées. Deux d'entre elles figurent des gallinacés, la dernière un chien ou un lion. Ce type d'agitateur à statuette zoomorphe est connu, sans être courant. On les retrouve majoritairement en contexte urbain.

Les objets les plus nombreux au sein de cette catégorie d'instrumentum mixte sont les petits instruments en alliage cuivreux, composés d'une tige munie aux extrémités de terminaisons diverses : sondes, spatules, cuillères, cuillerons. 


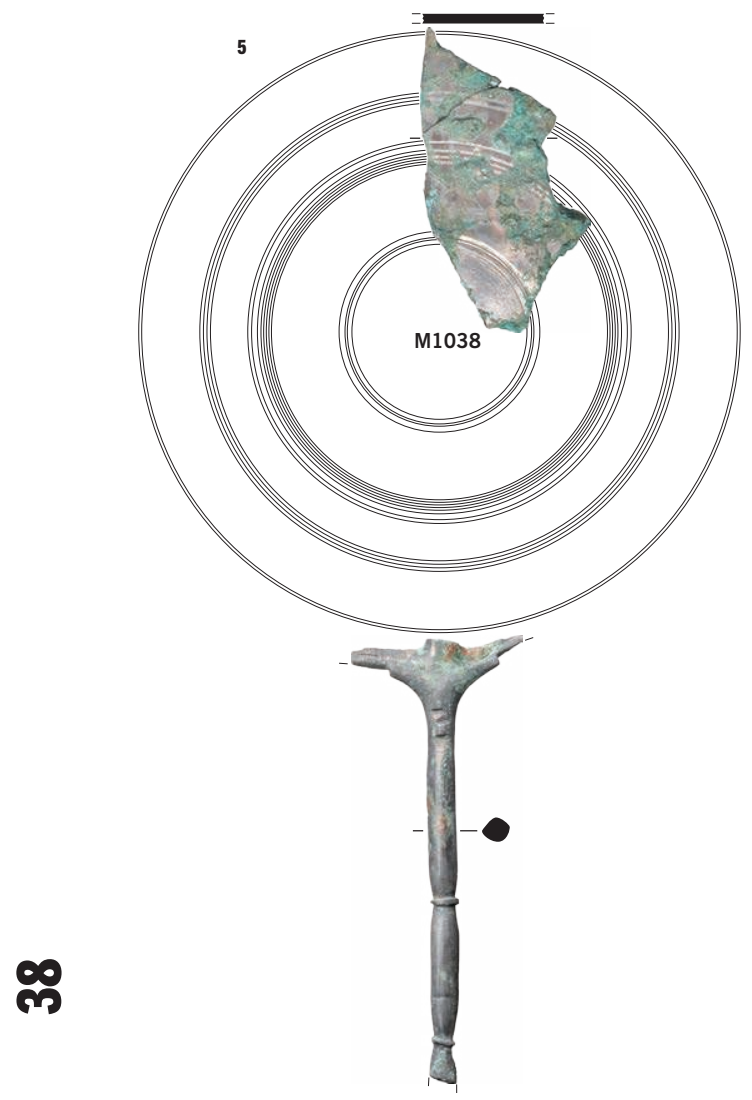

M1039

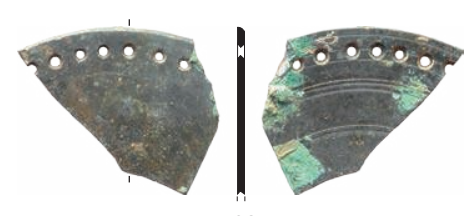

M285

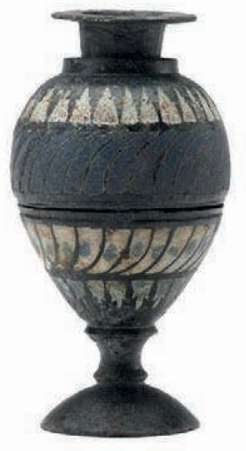

5. Sélection d'objets de toilette découverts à Famars :

fragments de miroirs,

manche, pincette à anneau do suspension et vase à parfum.

6. Ce nécessaire de toilette

népalais contemporain,

présentant un petit cure-

oreilles, une pincette et un

cure-ongles, tous en alliage

cuivreux et suspendus à un

anneau, est comparable aux

exemplaires antiques de

Famars.

7. (Page de droite) Sélection

d'objets d'usage mixte

découverts à Famars :

agitateurs, balsamaires,

tablette à broyer, cuillères-

sondes, sondes auriculaires,

pincettes.

8. (Page de gauche, bas)

Sélection d'objets médicaux

découverts à Famars :

possibles érignes, instrument à palette, sonde.
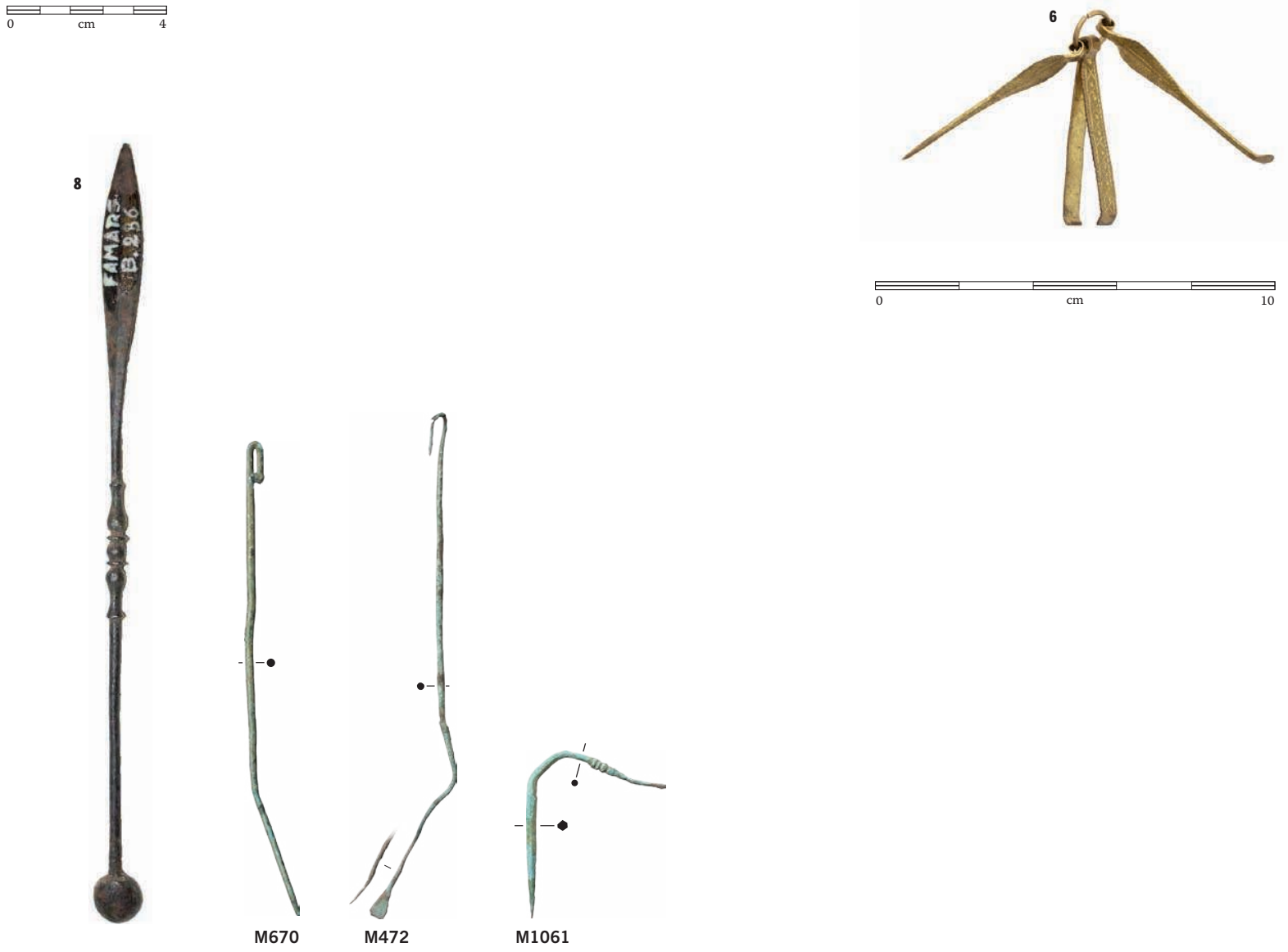


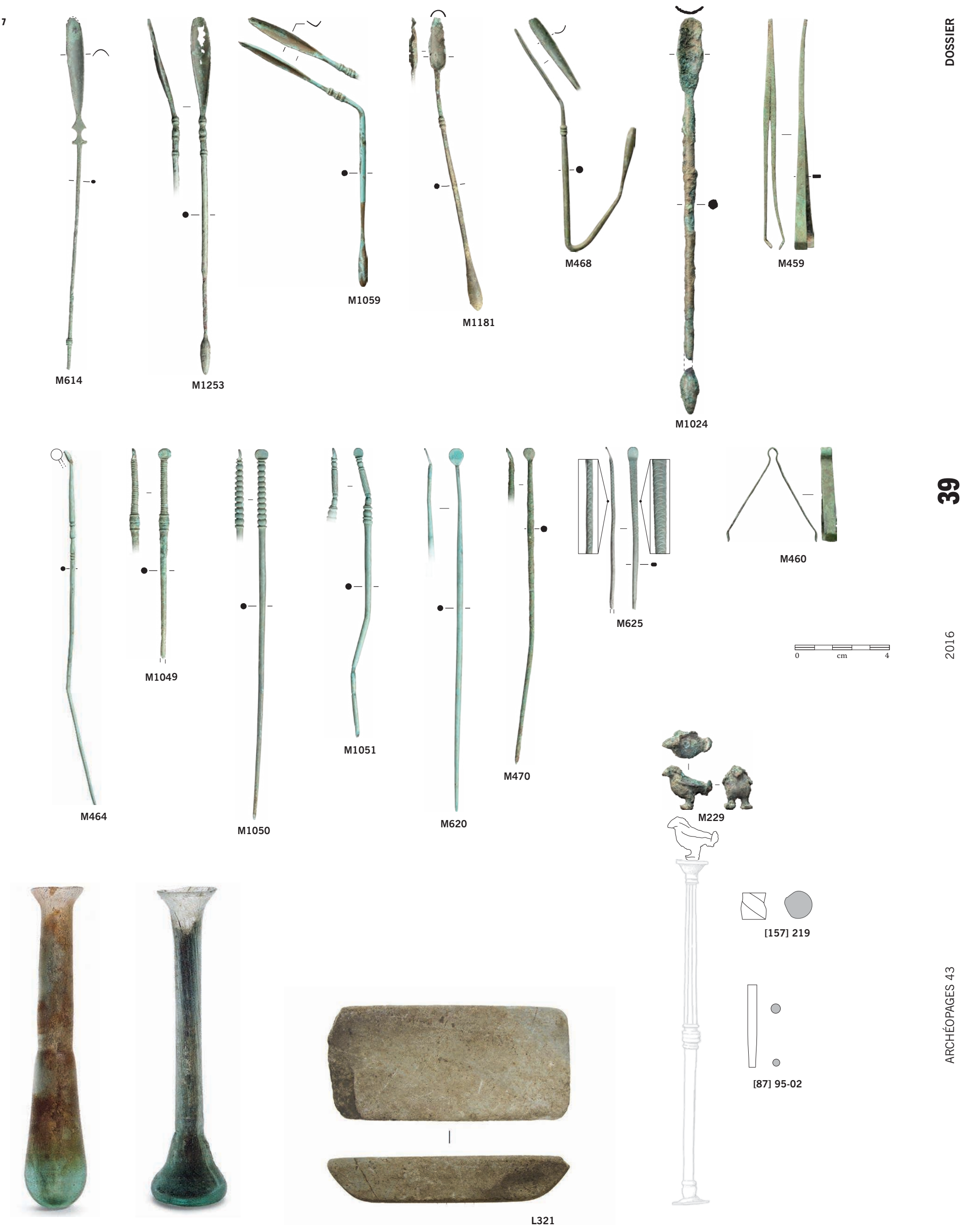


On compte ainsi 56 sondes auriculaires terminées par une extrémité pointue et par un petit cuilleron lenticulaire. Si certains [ill. 7, M-1049 et M-1051] présentent des analogies morphologiques avec des épingles du haut Moyen Âge, leur contexte de découverte assurément antique permet de les identifier comme des sondes auriculaires. Ces instruments, parfois nommés cure-oreilles, font encore l'objet de discussion quant à leurs possibles usages. Or, les textes latins et le recours à l'ethnographie apportent un certain nombre de réponses. On a vu plus haut que les cure-oreilles, de morphologie tout à fait analogue, sont encore employés de nos jours au Népal. Celse dans son Traité de la Médecine confirme l'utilisation de sonde auriculaire pour ôter le cérumen ou tout autre corps situé dans le conduit auditif : «Dès que quelqu'un commence à avoir l'ouïe un peu dure [...] ; la croûte se dissout ainsi elle-même, et se laisse extraire plus aisément avec la sonde auriculaire. [...]» (livre VII, chap. VII, $\$_{7}$ ).

Il semble donc que la fonction première de ces objets soit bien celle de nettoyer les oreilles, ce que confirme leur nombre élevé : il s'agit bien d'objets du quotidien. Mais on sait par ailleurs que ces objets pouvaient aussi être employés pour extraire des calculs urinaires ou réaliser des hémorrö̈dectomies. Ainsi, Celse nous précise-t-il quel instrument employer pour retirer un calcul urinaire : "Il faut, si c'est possible, le retirer à l'aide d'une sonde auriculaire ou de l'instrument qui sert, dans la taille, à extraire la pierre. [...] » (livre VII, chap. XXVI, \$2). Plus loin, il explique le traitement chirurgical des hémorroïdes (livre VII, chap. XXX, $\$ 3)$ : «Est-elle un peu volumineuse et à base un peu large, on l'accroche avec une ou deux petites érignes, et on l'incise légèrement au-dessus de sa base [...] résultat qui s'obtient en ne tirant ni trop ni trop peu les érignes. Puis, dans l'incision, on enfonce une aiguille au-dessous de laquelle on lie la petite tumeur avec un fil. [...]. Au bout de cinq ou six jours, on enlève la charpie avec une sonde auriculaire [...] ». On remarque à Famars une importante variété typologique des sondes auriculaires, basée sur les différents systèmes décoratifs du manche. Ainsi, si les exemplaires sans décor sont les plus nombreux et constituent le type le plus simple et le plus commun, on observe également des manches moulurés ou ornés d'incisions. Les exemplaires inornés ne sont pas caractéristiques du point de vue chronologique, mais ceux à manche décoré sont datés du Haut-Empire.

Outre les sondes auriculaires, on trouve des cuillères-sondes (21 NMI) terminées par une extrémité oblongue allongée en forme d'olive, la sonde, et de l'autre par un cuilleron fin allongé. Ces instruments étaient utilisés pour sonder des lésions dans un cadre médical, mais aussi pour écraser, mélanger et appliquer des onguents, qu'ils soient médicaux ou cosmétiques. Comme pour les sondes auriculaires, plusieurs variantes sont attestées : de la plus simple avec une moulure sur le manche destinée à faciliter la préhension à des types plus complexes, à manche facetté par exemple. Là encore, les exemplaires simples sont les plus nombreux et peu caractéristiques d'une époque, les autres variantes sont moins communes et leur datation est resserrée au Haut-Empire.

On compte également de rares sondes à spatules, terminées à la fois par une sonde oblongue et par une large spatule plate allongée. Leur utilisation est assez proche de celle des cuillères-sondes, la spatule servant ici sans doute à appliquer les préparations. Peu représentées à Famars, elles pouvaient répondre à un usage précis ou faisant doublon avec celui des autres sondes, ce qui expliquerait leur nombre peu élevé. Deux des trois exemplaires reconnus proviennent des fouilles anciennes et sont conservés aux Musées Royaux d'Art et d'Histoire de Bruxelles et leur morphologie les rattache aux $\mathrm{II}^{\mathrm{e}}$ et $\mathrm{III}^{\mathrm{e}}$ siècles de notre ère. Nous ne pouvons malheureusement pas savoir si ces deux objets faisaient ou non partie d'un même ensemble. Quelques tiges et sondes fragmentaires, appartenant à ces petits instruments composés d'une tige aux extrémités variées, complètent le lot sans que l'on puisse les identifier plus précisément.

Des pincettes de différentes dimensions pouvaient également être utilisées tant pour la toilette (épilation) que pour saisir des corps étrangers dans une plaie. Leurs usages sont donc multiples. Les variantes auxquelles elles appartiennent sont plutôt communes et peu caractéristiques d'une période, à l'exception d'un modèle à branches soudées dans leur tiers supérieur, daté de la seconde moitié du $\mathrm{I}^{\mathrm{er}}$ et du début du $\mathrm{II}^{\mathrm{e}}$ siècle de notre ère. Rappelons la découverte déjà citée d'une pincette appartenant à un nécessaire de toilette.

Enfin, la découverte de petites tablettes à broyer en pierre dure à grain fin, destinées à écraser des produits tant cosmétiques (fards par exemple) que médicaux, complète le panel des objets d'usage mixte. Une tablette soigneusement polie est marquée dans la partie centrale de sa face supérieure d'une légère dépression permettant d'éviter la dispersion des éléments à broyer et mélanger [ill. 7, L-321]. Une autre (L-1413), soigneusement polie elle aussi, présente sur sa face supérieure de nombreuses incisions indiquant un usage intense, ici de découpe et/ou d'affutage des instruments.

\section{Les objets à usage médical}

L'instrumentum médical est à la fois très faiblement représenté (5 NMI) et par des objets dont l'identification n'est pas toujours certaine [ill. 8]. Ainsi, on note la présence d'une possible érigne [ill. 8, M-670], un instrument destiné à écarter des tissus et terminé en crochet. Cette fonction peut également être reconnue sur un instrument terminé d'un côté par une palette et de l'autre par 
une extrémité recourbée [ill. 8, M-472]. Deux autres instruments à palette ont été intégrés aux objets médicaux par analogie formelle à M-472. Enfin, une sonde, aujourd'hui conservée aux Musées Royaux d'Art et d'Histoire de Bruxelles, terminée d'un côté par un large cuilleron circulaire et de l'autre par une spatule lancéolée (MB286-1) est un objet dont l'usage est purement médical. Sa découverte, ancienne, n'est malheureusement pas localisée au sein de l'agglomération, même si on peut supposer que cette sonde provient du secteur central de la ville, à savoir thermes et castrum, celui ayant fait l'objet des investigations les plus anciennes.

Ces quelques objets médicaux renvoient à des activités qui semblent sinon sporadiques, du moins très difficilement perceptibles en l'absence d'indices plus éloquents. Il est très difficile, voire impossible, de savoir pour quelles pathologies ces instruments étaient utilisés et leur faible proportion ( $3 \%$ du corpus) les rend presque anecdotiques. Pour comparaison, à Augst/Augusta Raurica, la capitale des Rauraques, les objets d'usage purement médical représentent 10,3\% du corpus des objets de soins, de toilette et de médecine (Riha, 1986). L'absence de tombes de médecin ou d'inscriptions mentionnant des médecins ou encore l'absence de cachet à collyre qui sont des éléments probants pour identifier une véritable présence de médecins place Famars, simple agglomération commerciale et religieuse, dans une position différente de celle des capitales de cité où les médecins étaient plus implantés. Par exemple, à Avenches/Aventicum, la capitale des Helvètes, une inscription est dédiée aux médecins et professeurs (Corpus Inscriptionum Latinarum, XIII, 5079) par deux dédicants dont l'un est médecin, ce que l'on sait par ailleurs grâce à des collyres portant son nom (Hirt, 2000, p. 97).

\section{L'importante représentation de la cosmétique par rapport à la médecine}

La plupart des types d'objets de toilette et de cosmétique recensés à Lyon (Vigier, 2015) sont attestés à Famars [ill. 9], à l'exception des peignes et des rasoirs. Nous avons déjà proposé que l'absence de ces derniers soit liée à la difficulté d'identifier des objets aux lames et tranchants fins. L'absence de peignes peut quant à elle trouver une explication d'ordre chronologique. Durant les trois premiers siècles de notre ère, le matériau privilégié pour les peignes est le bois, plus particulièrement le buis, avant d'être supplanté par l'os à la fin du III ${ }^{\mathrm{e}}$ siècle. On a déjà mentionné qu'aucun objet en bois n'avait été mis au jour à Famars, faute de milieux permettant leur conservation. Les peignes en os sont caractéristiques de l'Antiquité tardive. Or, la plupart des fouilles menées à Famars depuis quarante ans ont porté sur des secteurs de l'agglomération en déclin à partir de la fin du $\mathrm{III}^{\mathrm{e}}$ siècle, alors que l'occupation se resserre vers castrum dans les années 320. L'absence de fouille récente dans le centre de l'agglomération nous prive à la fois de données sur l'occupation du BasEmpire et d'informations précises sur l'occupation de la zone située vers les grands thermes.

La découverte de presque tout le panel des objets communs de toilette et de cosmétique à Famars tend à prouver que ces objets sont usuels. Concernant l'instrumentum d'usage mixte, tant pour la toilette et la cosmétique que pour des soins médicaux, on remarque que leur variété est elle aussi importante; neuf types d'objets sur les quinze répertoriés par Élise Vigier sont attestés, indiquant là aussi un usage courant. L'absence de certains types, tels que cuillère à parfum en os et strigile en os, fait peut-être écho à la faible représentation des objets en matières dures animales déjà soulignée. L'absence de mortier et de broyeur a, elle aussi, été mise en lien avec le matériau mis en ouvre - la pierre (voir supra). Comme attendu au vu du faible nombre d'objets, l'instrumentum purement médical résulte de pratiques difficilement perceptibles que l'on peut qualifier d'anecdotiques ou d'exceptionnelles.

On peut encore citer la découverte de plusieurs objets, non intégrés à notre corpus du fait de leur polyvalence, dont la fonction n'est pas directement de soigner ou prendre soin, mais qui peuvent être utilisés dans ce cadre et qui montrent à quel point les questions de soin, de santé et d'hygiène s'inscrivent dans un spectre large de la vie quotidienne. C'est le cas par exemple des cuillères en métal ou en matières dures d'origine animale ${ }^{7}$, qui peuvent tout à fait servir pour la préparation d'onguents, ou de deux petites balances à plateaux dont les dimensions les recommandent pour des petites quantités, pourquoi pas d'ingrédients pour préparer des onguents. D'autres objets attribués généralement à l'artisanat pourraient tout à fait être utilisés dans des activités médicales, comme un couteau à petite lame en quart de cercle servant à des découpes très précises ; une partie des aiguilles à chas pourraient avoir été utilisées en chirurgie de même que les lancettes, instruments composés d'un manche souvent torsadé et d'une lame lancéolée.

\section{La localisation des découvertes}

Pour les objets issus des fouilles récentes $\mathrm{du}$ Technopole, qui constituent près de $69 \% \mathrm{du}$ corpus, l'utilisation d'un SIG permet d'apporter nombre d'informations pertinentes quant à la place - au sens littéral ou figuré - qu'avaient les objets de toilette et de médecine au sein d'un quartier d'habitat à vocation artisanale de statut non privilégié en périphérie de l'agglomération. On observe ainsi deux phénomènes principaux, une large répartition des objets et quelques concentrations de mobilier intéressantes [ill. 10].

En s'affranchissant des données chronologiques dans la mesure où le phasage du site est en cours de réalisation, on observe tout d'abord une dispersion
7. Intégrées quant à elles aux études lyonnaises. le centre de la ville qui voit la construction d'un 


\section{$5 \mathrm{NMI} / 8 \mathrm{NR}$}

forces
miroir
strigile

vase à parfum

nécessaire de toilette

peigne

rasoir

en gras : objets attestés à Famars

en italique : objet attesté à Famars mais non intégré au corpus car d'usage médical incertain

\section{érigne}

instrument à palette

sonde médicale

scalpel

lancette?

cachet à collyre

ventouse

aiguille chirurgicale

pince à forcipressure

cautère

aiguille à cataracte

levier à os

compas

pince à castrer

speculum utérin ou rectal

autres
9. La plupart des objets de toilette représentés à Lyon

(Vigier, 2015) le sont aussi à Famars, les objets d'usage mixte sont également très variés alors que les objets à usage médical ne le sont pas. 10. Les objets de soin

découverts sur les fouilles du

Technopole sont répartis sur

l'ensemble de la fouille avec

quelques concentrations

ponctuelles.
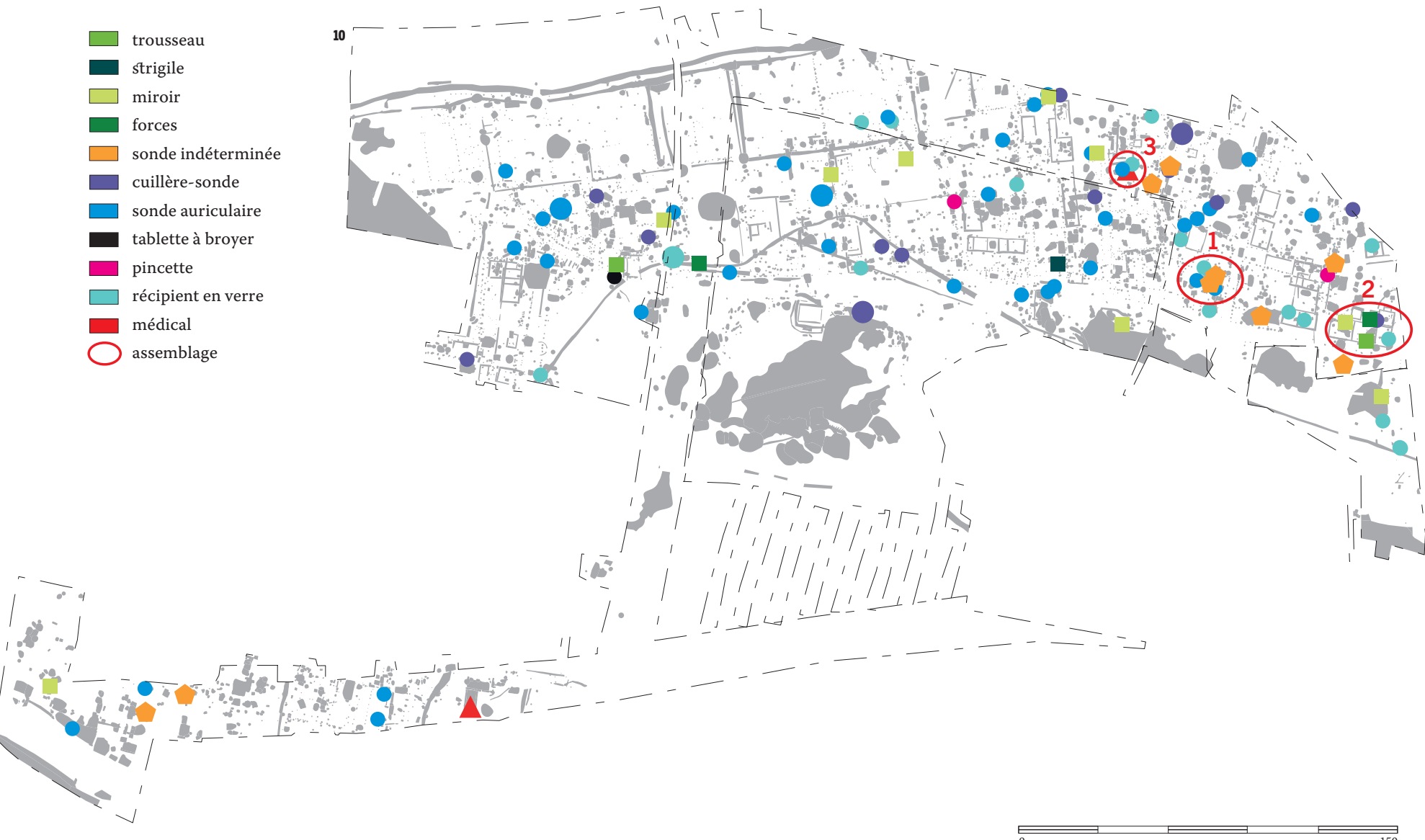
des objets dans les espaces d'habitat, les secteurs d'activités artisanales stricto sensu comme la zone d'extraction de matière première au centre de la fouille n'en livrent pas ou à la marge. À l'exception de ces espaces « vides », l'ensemble des parcelles d'habitat ou presque est concerné par la découverte d'objets de soin. En affinant le point de vue, on s'aperçoit que ces objets sont généralement trouvés à l'avant des parcelles, dans les zones bâties, et que l'arrière des parcelles en semble plus dépourvu. On perçoit même un effet d'alignement des trouvailles à l'avant des parcelles, parallèle à l'axe de la rue. La distribution des objets de soin sur toutes les parcelles d'habitat du Technopole atteste bien d'une pratique quotidienne de « l'hygiène » quel que soit le statut des populations. L'exemple des sondes auriculaires est le plus probant; d'autres objets comme les récipients en verre ou les cuillères-sondes sont également bien diffusés à l'ensemble des parcelles d'habitat.

Avec toute la prudence nécessaire à la lecture de cartes ne prenant pas en compte les paramètres chronologiques, on observe quelques concentrations d'objets remarquables. Ainsi, l'assemblage 1 regroupe cinq objets mixtes, à savoir deux sondes auriculaires et deux indéterminées ainsi qu'un récipient en verre. En l'état, rien ne permet de statuer sur ce regroupement d'objets qui semble curieusement situé en dehors des espaces domestiques. À l'inverse, on peut noter la présence dans un espace domestique (assemblage 2), d'un fragment de miroir, d'une pincette liée à un trousseau, d'une paire de petites forces, d'une cuillère-sonde et d'un récipient en verre. Dans ce cas, et au vu du contexte, on peut considérer que ces objets appartiennent bien à la sphère de la toilette et des cosmétiques et sont donc cohérents avec le contexte. Un dernier exemple qui pose question est celui d'un regroupement (assemblage 3 ) d'un récipient en verre, d'une sonde auriculaire et d'un possible instrument médical. Dans ce cas, on peut légitiment s'interroger sur la composante « médicale » de cet assemblage.

L'examen minutieux de ce corpus permet de rappeler que si le fait médical est difficile à mettre en évidence pour l'Antiquité, la santé dépendait également, comme de nos jours, d'une hygiène de vie qui, outre les aspects physiques et nutritionnels, passait par un ensemble de gestes quotidiens. Les fouilles du Technopole montrent qu'à Famars les instruments liés à l'hygiène se retrouvent sur la quasi-totalité des habitats. On peut donc supposer une pratique de soins quotidiens qui semble largement répandue et ne se cantonne pas aux espaces spécifiques comme les thermes. La difficulté, déjà soulignée, de la reconnaissance des instruments médicaux se heurte également à la polyvalence de certains objets tels les sondes auriculaires qui peuvent être utilisées tant dans leur fonction primaire que pour des actes chirurgicaux.
Références bibliographiques

Celse, Traité de médecine de A. C. Celse, traduction nouvelle, avec texte latin..., A. VÉDRÈNES (éd.), Paris, Masson, 1876, $797 \mathrm{p}$.

Corpus Inscriptionum Latinarum, XIII, HiRSCHFLED O., Zangemeister C. (éd.), Inscriptiones trium galliarum et germaniarum latinae, Berolini, apud W. de Gruyter, 1899-1943.

Pline l'Ancien, Histoire Naturelle, Livre XXXIIL, Zehnacker H. (éd.), Paris, Les Belles Lettres, 1999, $124 \mathrm{p}$.

Bertrand I., 2003, Objets de parure et de soins du corps d'époque romaine dans l'Est picton (Deux-Sèvres, Vienne), Chauvigny, Association des Publications Chauvinoises (« Mémoire des A.P.C. », XXIII), 223 p.

Chevrier S. (dir.), en cours, Saint-Clément, Yonne, Bourgogne. Structures domestiques protohistoriques et domus antique en périphérie de Sens. Un ensemble-clos d'objets métalliques agricoles gallo-romains exceptionnel découvert dans une cave, rapport d'opération, Inrap-SRA Bourgogne.

Clotuche R. (dir.), 2016, FANUM MARTIS : Une agglomération à vocation religieuse, commerciale et militaire à la frontière du territoire nervien. Le PAGUS FANOMARTENSIS, un terroir spécifique?, rapport de PCR, Inrap-SRA Nord-Picardie, 551 p.
Delmaire R. (dir.), 1996, Carte archéologique de la Gaule 59, Le Nord, Paris, Éditions de la Maison des Sciences de l'Homme, $497 \mathrm{p}$.

Gourevitch D., 2011, Pour une archéologie de la médecine romaine, Paris, De Boccard, $250 \mathrm{p}$.

Hirt M., 200o, « Les médecins à Avenches/Aventicum. Étude basée sur l'ensemble du matériel relatif aux médecins ", Bulletin Pro Aventico, n 42, p. 93-133.

MALlet F., 2009, « Le strigile en Gaule : objet utilitaire et vecteur de romanité. L'exemple du strigile en fer à décor damasquiné de la villa augustéenne des Champs-de-Choisy à Charny (Seine-et-Marne) », Gallia, t. 66, p. 113-152.

Riнa E., 1986, Römisches Toilettgerät und medizinische Instrumente aus Augst und Kaiseraugst, Augst, Römermuseum, 1986 («Forschungen in Ausgt », 6), $85 \mathrm{p}$.

VIGIER É., 2015, « Aperçu de l'instrumentum de toilette et de chirurgie à Lugdunum. Un état des données », in Raux S., Bertrand I., Feugère M. (dir.), Actualité de la recherche sur les mobiliers non céramiques de l'Antiquité et du haut Moyen Âge, actes de la tableronde européenne Instrumentum, Lyon, 18-2o oct. 2012, Montagnac / Chauvigny, Éditions Monique Mergoil / Association des Publications Chauvinoises (« Monographie Instrumentum », 51 / « Mémoire des A.P.C. », XLIX), p. 577-609. 